\title{
Organisation and design of regular field hospitals
}

\author{
MCM Bricknell
}

\begin{abstract}
The organisation and design of field hospitals within the British Army has been under review since the Gulf War. This paper discusses principles for the organisation and design of field hospitals and makes suggestions for further development. The paper describes the deployment of field hospitals from a 25 bed hospital troop up to a 200 bed field hospital.
\end{abstract}

Key words: military, hospitals, design.

\section{Introduction}

The organisation and design of field hospitals within the British Army has been under review since the Strategic Defence Review. A comprehensive study of the requirements for land based medical units behind the divisional rear boundary in a theatre of operations has been conducted by HQ LAND command. This has led to a careful examination of the function and organisation of field hospitals. The aim of this paper is to describe principles for the structure and design of deployable military hospitals in order to inform the readership of this Journal of current developments within the regular field hospitals. The content of this paper has been endorsed by the chain of command. The concepts described may have utility for field hospitals within the Territorial Army but these units are not yet resourced to deliver the capabilities described.

\section{Scope}

The proposals contained in this paper are based on the current establishment of regular field hospitals(1). This paper does not examine field hospital structures beyond 200 bed capability. Theatre or Role 4 level capabilities will not be considered. The capabilities listed in the HQ LAND Study are considered to be the requirement for regular field hospitals. A regular field hospital is to be:

a. capable of deploying, and redeploying, in order to conform to the supported division during manoeuvre warfare and able to function in a relatively adverse military environment. They must be capable of operating on a 'green field' site, and be modular to facilitate Task Organisation into smaller or larger hospitals.

b. capable of identifying those casualties who will probably survive further evacuation (with appropriate sustaining treatment) to rear hospitals so that they may concentrate their efforts on providing primary surgery to ensure that all seriously injured casualties who would otherwise die are operated upon in the forward hospital within 4 hours:

c. capable of sustaining themselves in the field.

The proposals contained in this paper must be seen as separate from the grouping of Field Surgical Teams within Medical Regiments at Role 2. This capability is designed to meet the 2 hour clinical timeline for resuscitative surgery, and is only viable when supported by a Role 3 facility located elsewhere in the CASEVAC chain to meet the 4 hour rule.

The function of field hospitals in the Nuclear Biological and Chemical (NBC) environment has also been under review. This led to the procurement of equipment specifically designed to meet such a threat. Although much of the developmental work has also informed the requirement for the field hospital operating in a non-NBC environment, this paper will not specifically consider NBC issues.

Ideally, field hospitals should be sited in buildings with existing infrastructure services. However it has been stated that regular field hospitals must be able to activate at full capability on a 'green field' site. This is the greatest challenge for field hospitals. Detailed pre-planning is required to ensure effective use of external logistic support (eg. engineers, pioneers, mobile bath and laundry units). It has been agreed that templates for field hospital shelter systems should be created in order to ensure that the Unit Equipment Table (UET) supports such deployment and to practice the logistic planning process. The field experience of all 3 regular field hospitals during 2000 has been reviewed in order to produce this paper.

\section{Basic Principles}

General Design. The overall design of the field hospital site should include the following 4 functional components: clinical services, command, administrative support and accommodation. The size of these components clearly depends on the overall capability of the complex. A schematic for the site design of a field hospital is shown at 


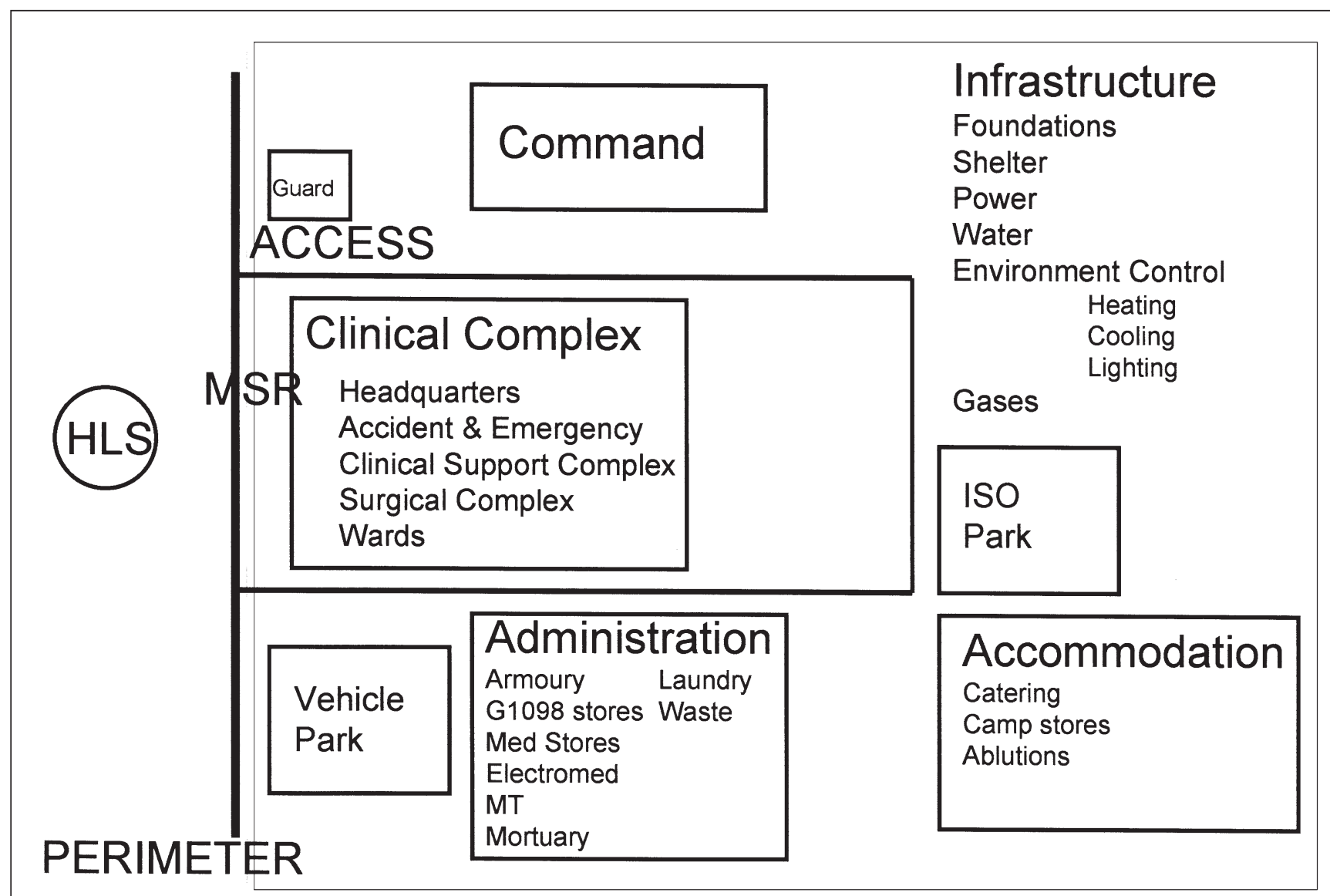

Fig 1. Schematic Field Hospital site.

Figure 1. If buildings or hard standing are available, this should be adapted to accommodate the field hospital. The designs contained in this paper assume the worst case scenario of a flat green field site.

Scales of Deployment. Field hospitals are equipped with 2 communications vehicles for operational deployment. This communications equipment allows field hospitals to be sub-divided into a maximum of 2 components: a main body ( $\max 150$ beds) and a single sub-unit (max 50 beds). The 'common user departments' (A+E complex, Evacuation and Support Sqn) all have a critical mass below which there is either a disproportional decrease in capability or the equipment reduction is illogical (e.g. removing a bay in resuscitation when there is space available in the allocated shelter). The basic division of these areas should be divided on a $75 \% / 25 \%$ template. Where the Medical Equipment Table (MET) provides for 3 modules (e.g. X-ray and laboratory) then these should be divided in $66 \% / 33 \%$ split. It is axiomatic that UET scales and the MET must be designed to support the same modular capabilities.

Command. There are 2 scales of command: Regimental Headquarters (RHQ) and a single Squadron Headquarters (SHQ). These are based on DRASH as the increasing use of computers for HQ functions requires robust protection from the weather. A $12 \times 12$ tent is included in each command group to function as a guard tent.

Regimental Headquarters (RHQ).

The RHQ has to be capable of managing a 'medical group' and/or a full 200 bed field hospital. Thus the RHQ is a large organisation which includes the Regimental Administrative Office and Medical Records Office functions. It is scaled for DRASH in order to provide a robust environment suitable for 24 hour working with sensitive IT equipment. The RHQ is separate from the clinical complex in order to avoid micromanagement by the command team. The design for RHQ is shown at Figure 2.

Squadron Headquarters (SHQ). The SHQ is a small command and communications (C2) node that commands the Hospital Squadron. It should work to a medical RHQ. This can be based on a single communications vehicle attached to a DRASH No 2.

Administrative Support. Administrative support to the field hospital consists of the following functions: Quartermasters department, Medical Stores Section, Motor Transport Section (including Light Aid Detachment), Catering Section, Medical and Dental Support Section and Artisan Section (Royal Engineer carpenter, electricians and plumber). These are scaled on a $150 / 50$ basis. The designs for administrative support require further development and are not included in this article.

Accommodation. The detailed 


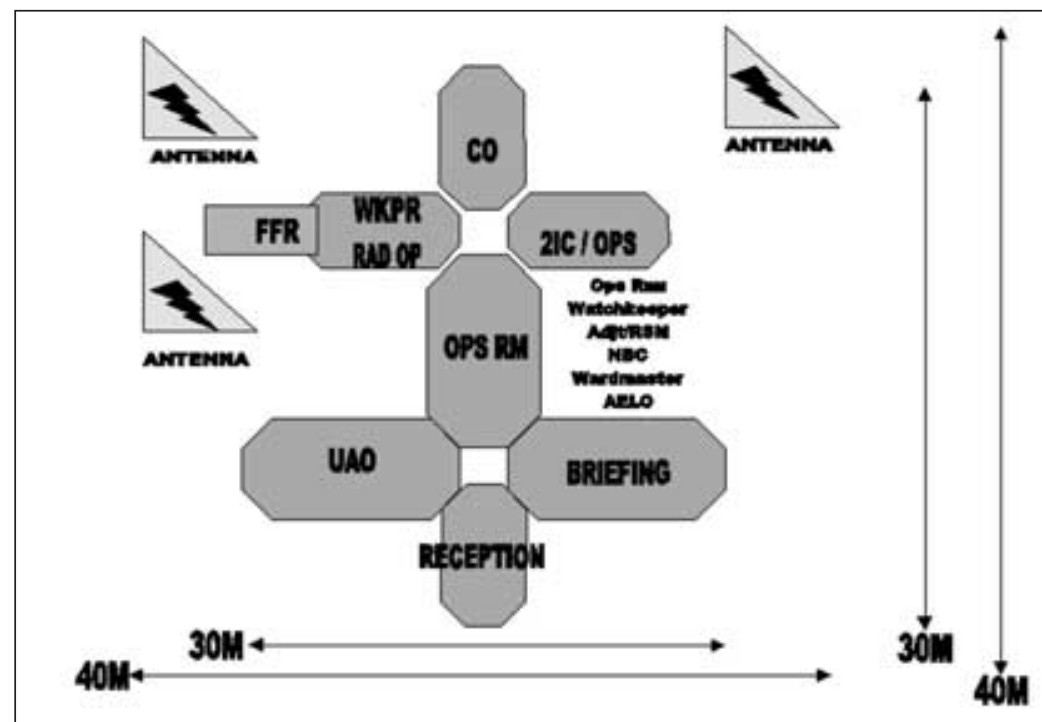

Fig 2. Regimental Headquarters. have two-way traffic in all but the smallest design and these shelters are too narrow to allow stretchers to pass each other. Therefore the spine should be composed of corridor pairs at above 50 beds of deployment. Unfortunately this creates a 'double apex' which drains water between the corridor pairs which may degrade the floor in the corridors in heavy rain. It might be necessary to add an additional 'vertical' spine at the end of each ward if the patient load generates congestion in the central spine. The ground occupancy of shelter systems is greater than the internal dimensions because of the necessity for guy lines. Thus it has been found necessary to have double corridors between ' 4 ways' to ensure sufficient space between lateral shelters. There must be sufficient external exits to enable convenient access to the complex and rapid exit in the event of fire. Neither the ' 4 way' nor corridor is provided with a groundsheet. This risks damage to the critical thoroughfare in the clinical complex. The ideal future design for the spine should be a single, two-way thoroughfare with a robust system for protection of the ground and specifically designed lateral connections to departments.

Shelter systems. The shelter system has the greatest influence over the clinical environment within which medical care is delivered. The main considerations are temperature control and cleanliness. The appearance of the clinical environment is also important to reinforce that they, the casualties have been removed from the chaos of the battlefield and are now safe(3). The current basic shelter system is GS tentage. GS tentage is provided in a variety of sizes. The most utilised size for the clinical complex is $18 \times 24 \mathrm{ft}$ that can be joined to construct $18 \times 48 \mathrm{ft}, 18 \times 72 \mathrm{ft}$ or $18 \mathrm{x} 96 \mathrm{ft}$ sized shelters. GS tentage is robust, simple and provides sufficient space for two rows of stretchers or beds. GS tentage is difficult to clean and may leak in heavy rain. The clinical environment may be improved by the addition of white liners. These also provide insulation and enhance the weather resistance of the GS tent. The DRASH shelter system is faster to erect, more weather proof and provides a better clinical environment compared to GS tentage. It is less robust and too narrow for two rows of stretchers or beds. An increasing number of military forces are using containerised facilities for key components of the hospital complex. The design of field hospitals should specify the shelter system for each department after full consideration of the merits of each system. Field trials have demonstrated that mobility of the X-ray equipment is seriously constrained if the groundsheet laid directly onto the ground. Every effort should be made to provide a 
flat surface onto which the ground sheets may be laid. Finally a sunshade may be erected over the entire complex to reduce the transmission of radiant heat into the clinical complex. The Improved Tented Camp shelter system may provide a suitable replacement for GS tentage for those departments that can be satisfactorily accommodated in soft-walled shelter systems.

Containerisation. Many military medical services have procured containerised systems for components of deployable field hospitals. The extent of this 'containerisation' varies but the operating theatres are most common department affected. Intensive Therapy Units, laboratories, imaging suites, and Central Sterile Supplies Departments are also containerised in some Armies. Whilst these may seem ideal systems, detailed engineering solutions are required to deliver an effective interface to soft-walled shelters, power and environmental control, lighting, and cold water systems.

UK is developing containerised facilities called the Mobile Transportable Surgical Facility (MTSF). This will be a major enhancement to the ability of the AMS to provide a standard of care as near to peacetime best practice as practicable(4). Once successfully fielded, there will need to be a detailed review of the design of field hospitals. It is suggested that:

a. When a field hospital deployment requires ISO containers then MTSF will normally be included. It may be necessary to deploy a limited Role 3 capability by air and thus at least 1 surgical team must be configured for this.

b. The 'resuscitation' shelter contains 4 bays. These are used: $2 \times$ Intensive Therapy Unit (ITU) beds and $2 \times$ High Dependancy Unit/recovery beds. The resuscitation function occurs within the $\mathrm{A}+\mathrm{E}$ complex.

c. At full scaling the MTSF will replace 2 surgical teams scaled to operate inside DRASH. The remainder will still need to function using DRASH.

\section{Clinical Functional Groupings} Accident + Emergency $(A+E)$ Complex. This area must include sufficient space for reception \& triage, treatments, resuscitation and an evacuation point (for return to duty (RTD) or evacuation without further treatment). At the 25 or 50 bed scale of deployment these functions can be contained in a single $18 \times 72$ GS tent. Above '50 beds' the functions should be contained into a T shape of $18 \times 72$ GS tents in separate departments. It should be noted that the Casualty Clearing Stations in World War 2 found it to be greatly advantageous to have an 'Evacuation' ward close to Reception to save unnecessary carrying of less serious cases through the complex. This is the 'Treatments' area that could also be used as an overflow for Reception in rush periods(5). Historical reports describe the function of a pre-operative ward in which serious casualties may be assessed by a surgeon and anaesthetist and resuscitated prior to surgery(6). This function will be undertaken in the resuscitation area of the $\mathrm{A}+\mathrm{E}$ complex.

Clinical Support Complex. The term 'Clinical Support Complex' has been chosen to encompass the specialist departments of laboratory, X-ray, physiotherapy, dental and psychiatry. These departments may be integral to the function of the field hospital or may be organised as assets within a theatre of operations. These are included as separate departments at ' 150 beds'. At ' 50 beds', the laboratory and X-ray have separate space but physiotherapy, dental and psychiatry are not included in the clinical complex. It is recommended that the laboratory and Xray departments should be placed before the surgical complex in the 'patient-flow'. This enables the services needed by the most urgent cases to be placed close to the resuscitation area of the A+E complex.

Surgical complex. The Surgical Complex must provide sufficient space for the functions of anaesthesia, surgery, intensive care (ITU), high dependency care (HDU), post-operative recovery, Central Sterile Supplies Department (CSSD), and theatre management. The critical care support should be routinely based on $2 \mathrm{x}$ ITU beds and $2 \times$ HDU beds per surgical table. The containerised systems such as the Modular Transportable Surgical Facility (MTSF) equipment must be the shelter system of choice. However the aspired scaling of 2 per regular field hospital only provides space for 2 surgical tables. The benefits of DRASH outway the restrictions in space compared to the environmental limitations of current GS tentage for an operating theatre. Therefore the remaining 4 general surgical teams and the burns team should be scaled to work in DRASH tentage. It is recommended that these should be scaled as pairs joined by a DRASH No 2 shelter. A single 'light' scaling in a DRASH No 6 is required for the final surgical team to support the Hospital Troop. It may be necessary to include this 'light' scale with all deployments of MTSF to provide redundancy should the utilities module fail. The reverse balance on choice of shelter applies to the ITU which should be placed in GS tents with white liners. The designs shown in the figures will be amended to include MTSF after the user trials have been completed.

Wards. The field hospital has been scaled 
around 25 bed wards. DRASH has been found to be too narrow for 2 rows of patients and thus there is insufficient nursing staff to manage 25 beds laid out in DRASH tentage. Therefore the wards should be built from GS tents with white liners. In order to provide sufficient space between beds, the ideal size of shelter for a ward is an $18 \times 96$, and a $12 \times 12$ (for the sluice area). Unfortunately trials by 22 Field Hospital has shown that the metal frame cannot sustain the weight of GS tentage and white liners at $18 \times 96$ size. The current choice is $18 \times 72$ with white liners (with limited but manageable space between beds) or $18 \times 96$ without. A ' 4 way' and 2 corridors are included in the Ward scale to enable a ward to join onto any field hospital design. Ideally patients should be taken directly from the ward to an evacuation vehicle. In periods of high casualty flow it may be necessary to hold patients after transfer from beds to stretchers and therefore an evacuation department is required. This should be sited so as to allow an ambulance to reverse up to the complex and reduce the exposure of the casualty to the weather.

\section{Capability Groupings}

The endorsed capability groupings are described below. These generic descriptions have been supported by detailed plans for use within units.

\section{Bed Hospital Troop (Hosp Tp)}

The Hosp $\mathrm{Tp}$ is designed to operate in support of a Role 2 medical unit (Medical Regiment dressing station) in order to provide a Role 3 capability. The Hosp Tp needs to integrate with the reception, treatment and evacuation functions of the host unit. This medical grouping may be used to support a $\mathrm{Bn} \mathrm{Gp}$ operation or to deploy early in the medical Desired Order of Arrival Staff Table (DOAST) of a theatre entry or theatre activation force. This grouping will compromise the mobility of the Role 2 unit and therefore the utility of this grouping may be limited in a warfighting senario. The Hosp Tp may be packed on pallets in order to be air portable. Thus these are light scales based solely on clinical capability and the Hosp Tp will require infrastructure support from host unit ( $\mathrm{C} 2$, food, water, accommodation etc). It also lacks integral lift and this should be grouped from another unit for the duration of deployment if the Role 2 is to retain mobility. The surgical shelter system is based on DRASH. The overall clinical capability of the Hosp Tp is: 1 surgical team, 2 ITU and $2 \mathrm{HDU} /$ recovery beds, laboratory, X-ray (including ultrasound) and a 25 bed ward. This is shown in Figure 3.

\section{Bed Hospital Squadron (Hosp Sqn)}

The Hosp Sqn is designed as an autonomous sub-unit that might be commanded by a RHQ (most probably that of a Medical Regiment) as part of a medical group. It contains the full range of Role 3 clinical functions by reinforcing the Hosp $\mathrm{Tp}$ with an $\mathrm{A}+\mathrm{E}$ Complex and evacuation (at $25 \%$ of the Field hospital scaled capability). It is self-sustaining and is capable of establishing at an independent location. The deployment of the Hosp Sqn is based on ISO containers due to the size of the support troop required. Therefore MTSF should be utilised as the surgical shelter system. The sub-unit lacks integral lift and this should be grouped for the duration of deployment if mobility is required. This is shown in Figure 4.

\section{Bed Hospital}

The 50 Bed Hospital is the smallest independent element of a field hospital. It is

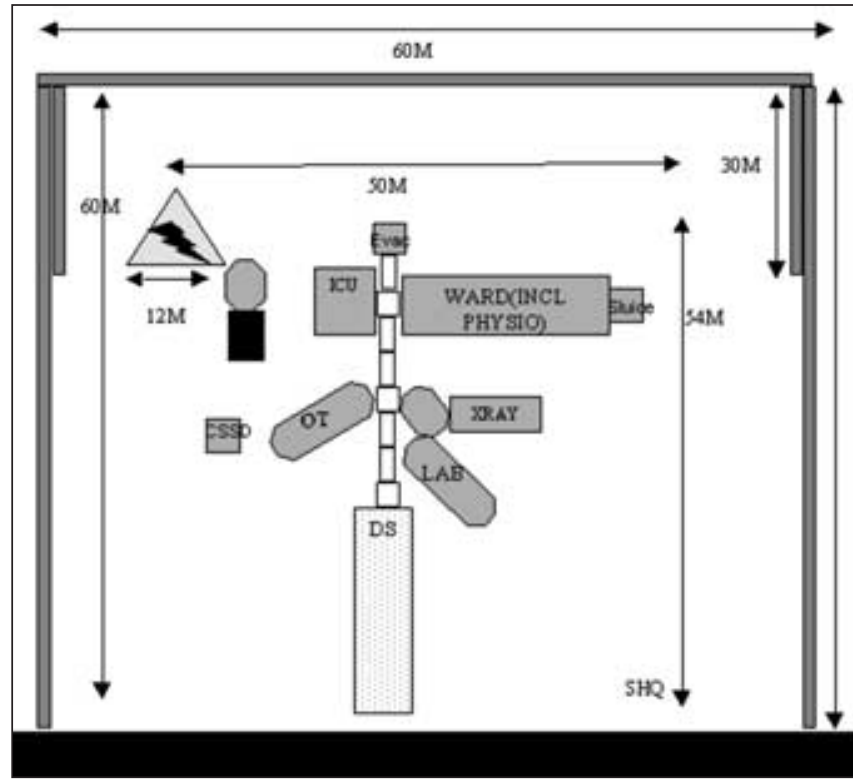

Fig 3. Hosp Tp (25 Beds).

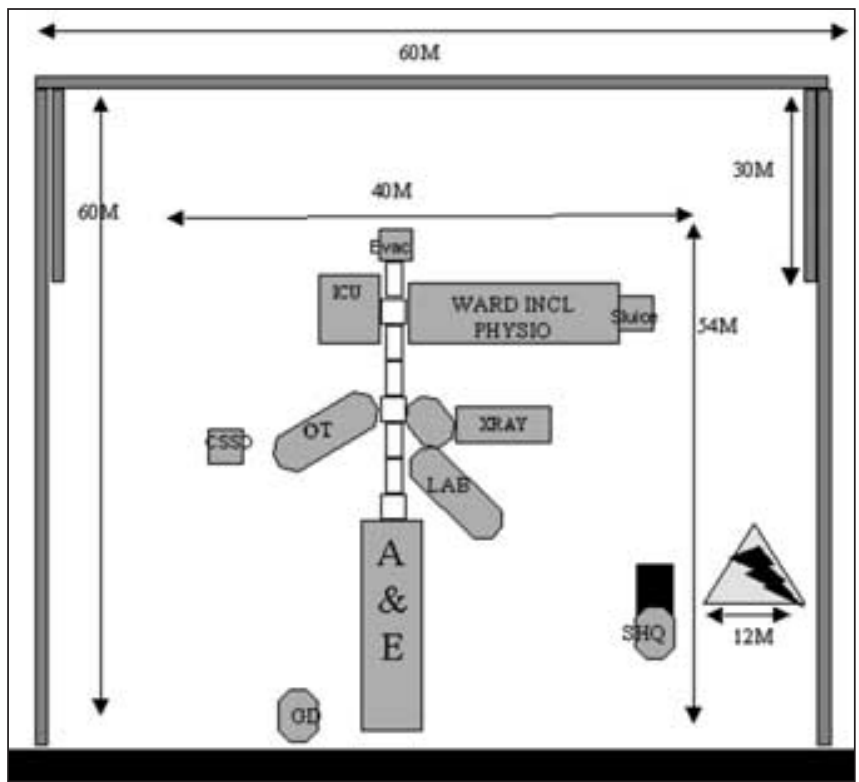

Fig 4. Hosp Sqn $(25 \mathrm{Bed})$. 


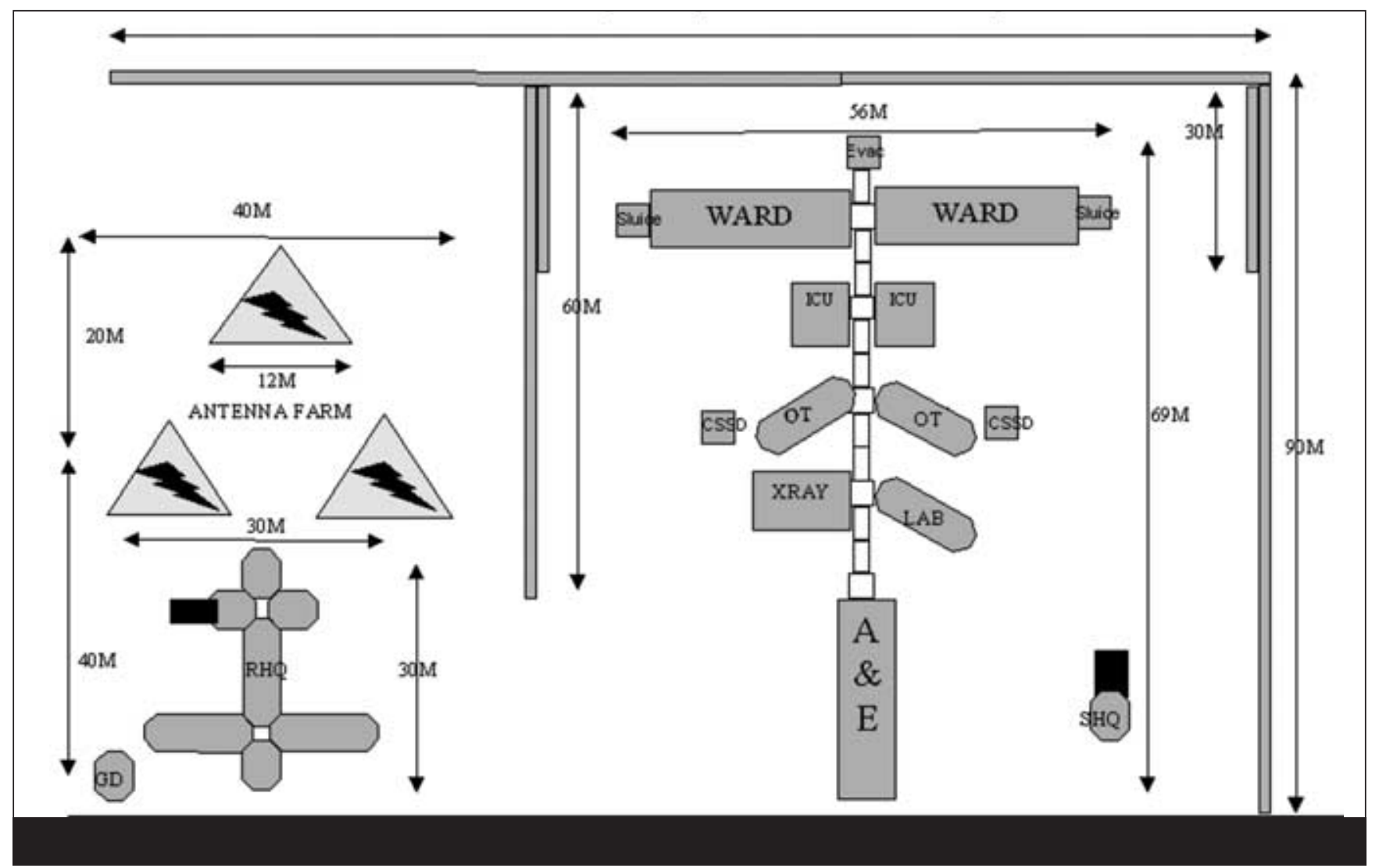

Fig 5. Hosp (50 Bed).

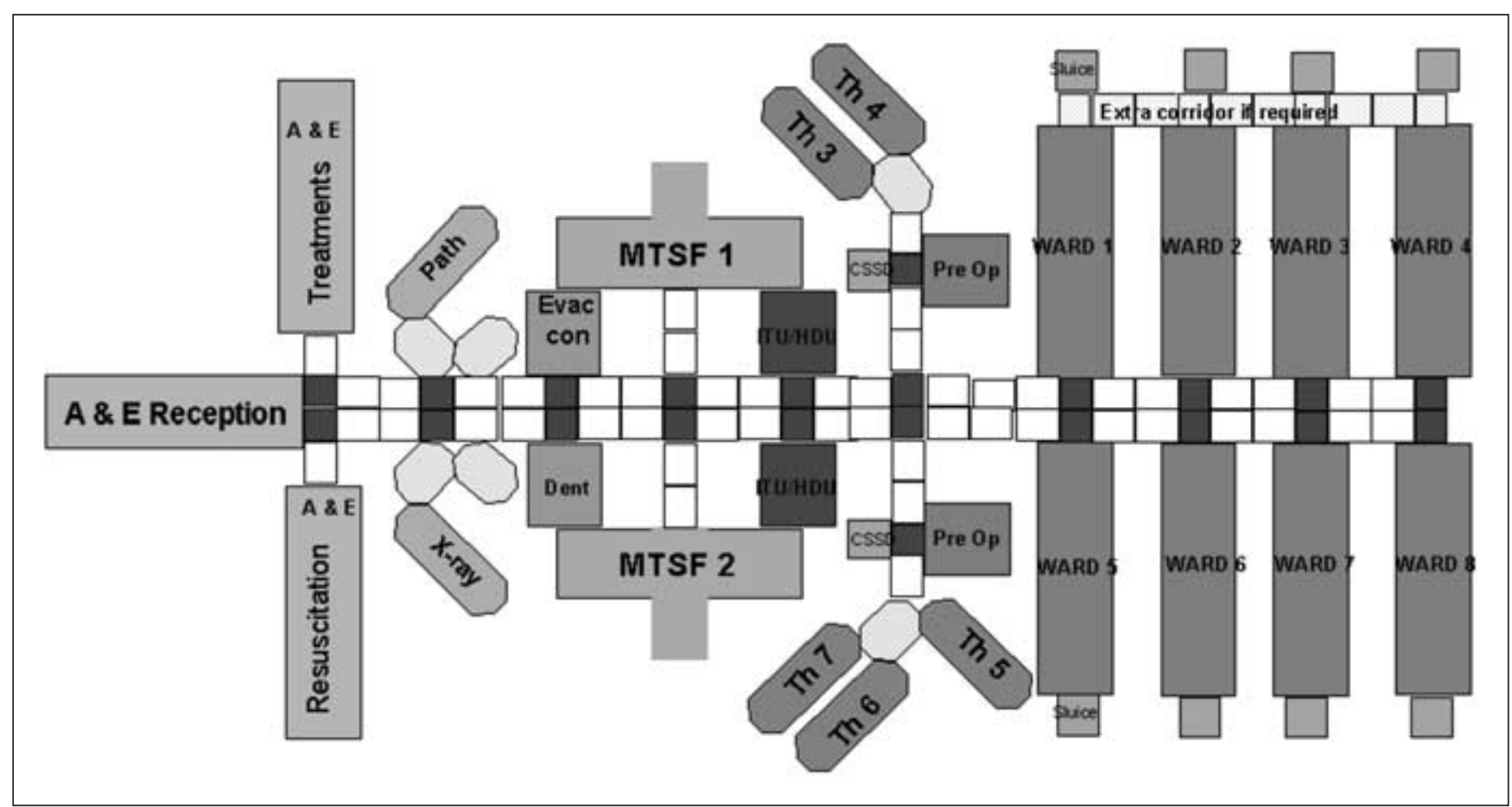

Fig 6. Hosp (200 Bed).

completely self-contained for C2 and infrastructure support. This includes the deployment of the RHQ and is able to take other medical sub-units under command. The Hosp Sqn clinical capability is augmented with a second operating theatre, ITU/HDU suite and a second further 25 bed ward. The size of a 50 bed hospital makes it unlikely to be suitable for a sub-unit level of command unless it has been detached after deployment of the full 200 bed capability. It lacks integral lift and will require external assistance for deployment and redeployment. External sub-units such as a GD section, band and mobile bath and laundry may be grouped with the unit. The surgical shelter systems would usually be based around two modules of MTSF. This is shown in Figure 5. 


\section{Bed Hospital and 200 Bed \\ Hospital}

The designs for the 100 Bed Hospital (4 surgical teams and 4 wards) and 200 Bed Hospital ( 7 surgical teams and 8 wards) are very similar except for the number of surgical teams and wards. The 'commonuser' areas are similar at both levels of deployment (based on $75 \%$ of total scale). At 200 beds the 50 bed capability from the 'common-user' area may be subsumed into the complex or remain separated in order to maintain the ability to detach a sub-unit. The design for the 200 bed hospital is shown in Figure 6.

\section{Summary}

This paper describes as series of principles for the organisation, design and deployment of regular field hospitals. A series of designs for each scale of field hospital deployment are shown in Figures $1-6$.

\section{References:}

1. AF C7005 dated 1 Apr 00 endorsed LAND 220/99 and ASD2C 57999.

2. US Army Field Manual 10-14 Employment of the Combat Support Hospital Tactics, Techniques and Procedures.

3. Army Doctrine Publication Volume 3 Logistics Medical Supplement May 2000.

4. NATO Medical support principles and policies. NATO MC 326/1 dated 14 Jun 99.

5. ED FAE Crew Medical History of the Second World War. Army Medical Services Campaign North West Europe. Volume IV. HMSO London 1962.

6. Fiddes FS. Surgery at the Casualty Clearing Station. F Roy Army Med Corps 1945; 84: 276-280. 